PAPER

\title{
Hippocampal size and memory function in the ninth and tenth decades of life: the Sydney Older Persons Study
}

\author{
T C Lye, O Piguet, D A Grayson, H Creasey, L J Ridley, H P Bennett, G A Broe
}

See Editorial Commentary, p 527

J Neurol Neurosurg Psychiatry 2004;75:548-554. doi: 10.1136/jnnp.2003.010223

See end of article for authors' affiliations .....................

Correspondence to: Dr T Lye, Centre for Education and Research on Ageing, Concord Hospital C25, Concord NSW 2139, Australia; tye@medicine.usyd. edu.au

Received 5 January 2003 In revised form

25 August 2003

Accepted

9 September 2003
Objectives: The purpose of this study was to define magnetic resonance imaging (MRI) correlates of normal brain ageing, with the specific objective of investigating whether the size of the hippocampus is selectively correlated with age related memory performance in non-demented individuals in their ninth and tenth decades of life.

Methods: Hippocampal size was estimated using MRI based volumetry and qualitative visual assessment in 102 community dwelling individuals aged between 81 and 94 years. Participants were evaluated on a variety of clinical and experimental instruments, including a comprehensive neuropsychological test battery. All participants underwent neurological examination, an extensive medical history was obtained, and an informant confirmed details of each participant's functional ability.

Results: Both visual and volumetric hippocampal measures were identified as robust predictors of memory performance, even when the influence of age related illnesses and sociodemographic variables was accounted for. When the sample was reduced to include the most cognitively healthy participants who were rated by an informant as showing no evidence of cognitive decline, the left hippocampal measures remained significant predictors of delayed retention of verbal material.

Conclusions: These findings suggest that hippocampal volumes are selectively correlated with memory functioning in both normal and successful ageing.
$\mathrm{K}$ nowledge and understanding of the role of the hippocampus in memory processing has greatly increased since the first clear demonstration of severe memory dysfunction following bilateral hippocampal resection. ${ }^{1}$ Further support for the association between the hippocampus and memory performance has come from neuroimaging studies of temporal lobe epilepsy, ${ }^{23}$ normal ageing, ${ }^{4}$ Alzheimer's disease, ${ }^{56}$ and head injury. ${ }^{7}$ Despite an abundance of literature documenting the pattern of cognitive decline and neuropathology associated with age related brain diseases such as dementia, until recently there has been a relative paucity of research effort directed toward identifying the non-pathological cognitive and structural brain changes associated with advancing age in neurologically healthy seniors. The purpose of this study was to investigate the relationship between magnetic resonance imaging (MRI) based visual and volumetric measures of hippocampal size and memory performance in community dwelling individuals over 80 years of age.

\section{METHODS}

\section{Subjects}

The participants were survivors of a random population study of older people living in central Sydney, Australia (the Sydney Older Persons Study). The initial sample consisted of 630 community dwelling subjects aged 75 years and over at the time of recruitment in 1991-1993, with approximately equal numbers of males and females. The method of sample selection has been described in detail elsewhere. ${ }^{8}$

At the 6 year follow up, all surviving participants still living independently in the community and capable of giving informed consent were invited to take part in this study. Owing to the nature of the MRI scanning process, individuals with either metallic inclusions (such as cardiac pacemaker) or claustrophobia were excluded on technical grounds. Individuals with a range of pre-existing illnesses that commonly occur in this age group were not excluded, although the nature of any medical condition was documented. Efforts were made to avoid applying overly stringent exclusion criteria that would result in an artificially healthy sample of older persons, as it was intended that the population based sample under study would provide insight into the relationship between hippocampal size and memory in very elderly community dwellers.

Neuropsychological data were collected from 123 individuals. Five did not undergo MRI scanning owing to claustrophobia, and 16 scans could not be analysed owing to poor scan quality or missing sequences, reducing the number of participants to 102. All subjects gave informed written consent to participate in the study. The study was approved by the Central Sydney Area Health Service ethics review committee.

\section{Procedure}

Participants were evaluated on a variety of cognitive and medical measures. These included a comprehensive neuropsychological test battery, the Clinical Dementia Rating (CDR) scale, ${ }^{10}$ neurological examination, and cerebral MRI scanning. An extensive medical history was also obtained from an informant to verify details supplied by the participant. Although the neuropsychological battery administered assessed all major cognitive areas, only the neuropsychological results from the memory domain will be discussed in the current paper. The memory tasks administered included the logical memory (LM) and visual reproduction (VR) subtests from the Revised Wechsler Memory Scale, ${ }^{11}$ and the California Verbal Learning Test (CVLT) ${ }^{12}$

\footnotetext{
Abbreviations: CDR, Clinical Dementia Rating; CES-D, Centre for Epidemiological Studies Depression scale; CVLT, California Verbal Learning Test; MRI, magnetic resonance imaging
} 


\section{MRI acquisition}

MRI scanning was performed on a $1.5 \mathrm{~T}$ scanner (Signa, General Electric Medical Systems, Milwaukee, WI, USA). Measurements of the hippocampus and whole brain were derived from a Tl weighted three dimensional fast spoiled gradient echo sequence performed in the coronal plane. This technique generated 124 contiguous $1.5 \mathrm{~mm}$ thick slices, using a repetition time of $12 \mathrm{~ms}$, echo time of $3.5 \mathrm{~ms}$, a $22 \mathrm{~cm}$ field of view, $30^{\circ}$ flip angle, and a matrix size of $256 \times 256$. Ratings of periventricular and deep white matter lesions were performed on hard copies of two axial sequences: $(a)$ an FSE2 sequence (TR/TE 3600/108 ms) with $5 \mathrm{~mm}$ thick slices, a $2.5 \mathrm{~mm}$ gap, a $22 \mathrm{~cm}$ field of view and a $256 \times 256$ matrix; and (b) a FLAIR sequence (TR/TE $10000 / 140 \mathrm{~ms}$ ) with contiguous $4 \mathrm{~mm}$ thick slices, a $22 \mathrm{~cm}$ field of view and a $256 \times 256$ matrix.

\section{MRI image processing}

The volumetric measurements were performed using the Analyze PC AVW software package (version 3.0; Biomedical Imaging Resource, Mayo Foundation, Rochester, MN, USA). Image processing was undertaken by trained raters who were blinded to all clinical data.

The hippocampus was defined using an established tracing protocol, the details of which have been published elsewhere. ${ }^{13}$ The hippocampal boundaries were manually outlined on 35-40 sequential coronal slices. The volume of the region was then automatically calculated by counting voxels within the threshold range. All hippocampal volumetrics were performed by the same trained rater (TCL). Intra-rater reliability (intra-class correlation coefficient) for repeated tracing in 19 subjects was 0.98 for the left and 0.96 for the right hippocampus.

Hippocampal volumes were corrected for inter-subject variation in head size by dividing the left and right hippocampal volume by a measure of whole brain volume. Whole brain volumes were derived using a semi-automated technique that relied upon thresholding to enhance boundary definition. This measure was obtained by separating the cerebrum from the overlying dura, CSF, and skull, and excluding portions of the brain stem below the medulla. Such tracings were performed on every third coronal slice throughout the brain, as preliminary analyses indicated that this technique would maintain accuracy and reliability. Whole brain tracings were performed by one of two trained raters who each performed $50 \%$ of these ratings. Tracing 32 brains a second time gave rise to a test-retest reliability coefficient of 0.97 .

Visual ratings of hippocampal size were undertaken by an experienced, fully qualified radiologist (LJR) who was not involved in the volumetric measurements, and who was blinded to the demographic and cognitive characteristics of the participants. For consistency, ratings were performed on the same coronal sequence that was used for the volumetric measures. Efforts were made to present the images for rating in a configuration similar to that used in everyday clinical practice in order to ensure that the results were clinically relevant. As such, every third coronal slice throughout the length of the hippocampus was displayed simultaneously on a 21 inch monitor in a manner resembling a typical radiological film with 12 images. In keeping with other visual assessment methods in the literature, ${ }^{14}$ ratings were made on a 4 point scale. As demonstrated by the examples provided in fig 1 , the orientation of the ratings was consistent with the volumetric measures, with a higher number indicating a larger hippocampus $(4=$ normal hippocampus, $3=$ mild atrophy, 2 =moderate atrophy, and $1=$ severe atrophy).
Periventricular hyperintensities and white matter abnormalities were rated by the same radiologist (LJR) using a technique that has been described in detail elsewhere. ${ }^{15} 16$ Briefly, the number and size of the lesions were rated using existing semiquantitative scales. ${ }^{17}{ }^{18}$ Periventricular hyperintensities were recorded (in $\mathrm{mm}$ ) in the frontal caps, lateral bands, and occipital caps. Deep white matter lesions were rated in the frontal, temporal, occipital, and parietal regions, and the number of lesions was recorded separately in each region on the basis of four different size categories $(3 \mathrm{~mm}$ or less, $4 \mathrm{~mm}$ to $10 \mathrm{~mm},>10 \mathrm{~mm}$, or large confluent lesion or infarct).

\section{Statistical methods}

The dependent variables were per cent retention on LM, per cent retention on VR, and total learning and per cent retention scores on CVLT. The hippocampal measures (volumetric and visual ratings), sociodemographic variables, and medical variables associated with common age related illnesses were identified as predictor variables. The sociodemographic variables included sex, age, years of education, and estimated IQ (as measured by the number of errors on the National Adult Reading Test version 2). ${ }^{19}$ The medical variables used as covariates were classified as extrapyramidal measures (timed walk, finger tapping performance, and a global extrapyramidal measure), vascular measures (clinical and objective arteriopathy measures, history of stroke/TIA, white matter abnormalities or periventricular hyperintensities on MRI, and usage of aspirin or anti-platelet agents), or "other" variables that may impact upon a participant's current level of cognitive functioning.

The arteriopathy measures and the global extrapyramidal measure have been defined previously by Waite et al. ${ }^{9}$ The MRI white matter ratings were undertaken as a central component of a separate study carried out on the same sample. ${ }^{15}$ However, in view of previously reported associations between white matter abnormalities and poorer memory performance in normal ageing, ${ }^{20}$ these measures were also included in the current analyses as markers for possible cerebral and other vascular pathology that could adversely affect cognitive performance. The "other" variables with potential impact on current functioning included the number of medications taken, score on the Centre for Epidemiological Studies Depression scale (CES-D), ${ }^{21}$ life satisfaction rating on a self report scale, ${ }^{22}$ informant ratings of the participants' response drive and behavioural control (such as apathy and impulsivity), ${ }^{23}$ and measures of hearing and visual impairment.

The independent effect of the various predictors on each of the memory performance dependent variables was examined using multiple linear regression. Firstly, univariate analyses exploring the association between hippocampal measures and a given memory measure were performed. The associations between the memory measure and the sociodemographic and medical predictors were also initially examined univariately. Subsequently, all predictors that were found to be univariately significant from the hippocampal, sociodemographic, and medical predictors were then simultaneously entered into a multivariate model in order to ascertain the unique contribution these variables make to performance on LM, VR, and CVLT.

All reported $\mathrm{p}$ values are rounded to three decimal places; thus $\mathrm{p}=0.000$ indicates that $\mathrm{p}<0.0005$.

\section{Memory function in "successful" ageing}

While the population based nature of the study group provided insight into the functional status of very elderly persons living in the community, it may be argued that the lack of stringent exclusion criteria may have failed to exclude 

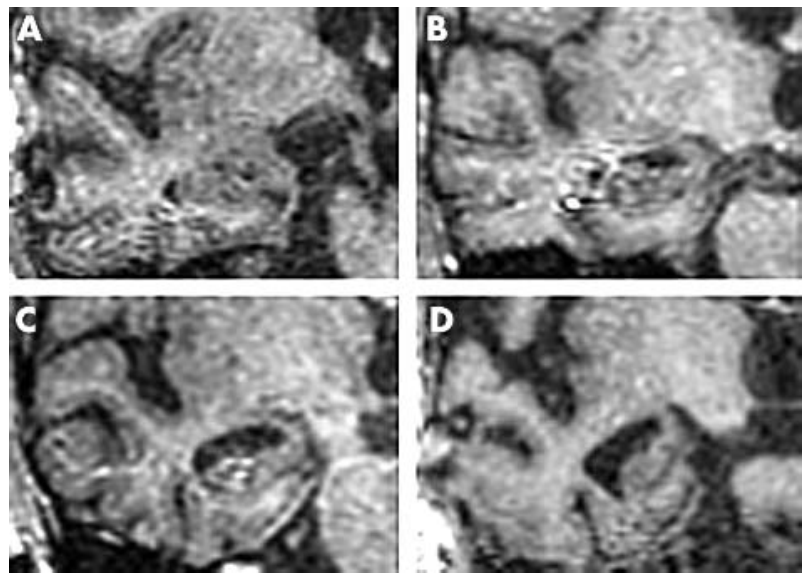

Figure 1 Coronal $\mathrm{T} 1$ weighted images through the medial temporal lobe demonstrating examples of the visual hippocampal ratings 4 point scale: (A) normal hippocampus (rating $=4$ ); $(B)$ mild hippocampal atrophy (rating $=3$ ); $(C)$ moderate hippocampal atrophy (rating =2); and (D) severe hippocampal atrophy (rating $=1$ ). The images are limited to the hippocampal subregion to enhance visualisation; however, the entire coronal slice was visible when the original ratings were performed.

some individuals who have experienced some degree of cognitive decline. Indeed, a small number of individuals included in this study had demonstrated some degree of cognitive change over the previous 3 years according to their informant rating on the CDR. This measure has been shown to be a valid instrument for the screening and staging of dementia in an earlier phase of the Sydney Older Persons Study ${ }^{24}$.

After identification of the predictors of memory functioning in the whole sample, the most cognitively healthy participants with $C D R=0$ were selected, and the data were then re-examined in these 57 individuals who had not demonstrated any evidence of cognitive decline according to their informant. This was performed to provide insight into the relationship between memory and hippocampal size in "successful" ageing. Of the non-selected participants, 34 were classified as demonstrating "questionable dementia" $(\mathrm{CDR}=0.5)$, six were rated as exhibiting "mild dementia" $(\mathrm{CDR}=1)$, while one individual was classified as having "severe dementia" (CDR =3). Four individuals did not have an informant, and therefore could not be assigned a CDR rating.

Table 1 Demographic and memory data on the whole sample and the reduced sample of the most cognitive healthy participants

\begin{tabular}{lll}
\hline Variable & $\begin{array}{l}\text { Whole sample } \\
(\mathbf{n}=102)\end{array}$ & $\begin{array}{l}\text { Reduced sample with } \\
\mathrm{CDR}=\mathbf{0}(\mathbf{n}=57)\end{array}$ \\
\hline Sex M:F & $54: 48$ & $28: 29$ \\
Age & $85.3(2.9)$ & $85.1(2.7)$ \\
Education (years) & $10.3(2.1)$ & $10.4(2.2)$ \\
Estimated full scale IQ & $109.0(9.9)$ & $110.4(9.7)$ \\
MMSE total score & $27.0(2.6)$ & $27.6(1.9)$ \\
Logical memory (\% retention) & $69.9(23.4)$ & $74.8(19.1)$ \\
Visual reproduction & $56.5(28.0)$ & $64.4(25.6)$ \\
(\% retention) & & \\
CVLT (\% retention) & $68.0(30.2)$ & $77.2(24.0)$ \\
CVLT total learning & $35.7(11.0)$ & $39.7(9.3)$ \\
(trials 1-5) & &
\end{tabular}

Other than for sex, results are mean (SD).

MMSE, Mini Mental State Examination; CVLT, California Verbal Learning Test.
Table 2 Mean hippocampal volumes and visual ratings across the age spectrum studied

\begin{tabular}{ccccclll}
\hline & & \multicolumn{2}{l}{ Hipp volume } & & \multicolumn{2}{l}{ Visual rating } \\
\cline { 6 - 7 } Age & $\mathbf{n}$ & Left & Right & & Left & Right \\
\hline 82 & 24 & 30.10 & 31.73 & & 3.02 & 3.04 \\
84 & 28 & 29.99 & 30.93 & & 2.61 & 2.66 \\
86 & 22 & 29.24 & 29.46 & & 2.73 & 2.68 \\
88 & 13 & 31.62 & 31.83 & & 2.58 & 2.62 \\
90 & 9 & 30.63 & 31.07 & & 2.11 & 2.17 \\
92 & 5 & 29.63 & 29.89 & & 2.70 & 2.70 \\
94 & 1 & 31.69 & 29.91 & & 1.00 & 1.00 \\
\hline
\end{tabular}

Hipp, hippocampal.

\section{RESULTS}

The demographic characteristics for the whole sample and the 57 individuals with no evidence of cognitive decline are presented in table 1.

The distribution of hippocampal size across the age spectrum under investigation is summarised in table 2 for the whole sample. To assist interpretation, age was transformed from a continuous variable into a series of age groups, each spanning a 2 year period. The association between age and hippocampal size was not a simple linear relationship, although the lack of any clear trends should be interpreted with caution because of the small number of cases in the older age groups.

\section{Hippocampal size and memory performance in normal ageing}

The unique influence of the significant univariate predictors on memory task performance was examined by entering these variables simultaneously into a multivariate model. The final multivariate models for each of the memory measures are presented in tables 3 and 4 . For ease of reading, predictors that became non-significant on all memory measures following the univariate analyses were excluded from the tables.

Two multivariate models were constructed for LM per cent retention: hippocampal volumes were entered in one, and visual ratings of the hippocampus in the other. All significant univariate sociodemographic and medical predictors were also entered into the models. The most salient feature of the LM models was that the left hippocampus made a significant contribution to explaining the variance on this measure, with larger hippocampi predicting superior memory performance. In contrast, the right hippocampal measures (volumetric and visual) were rendered non-significant when examined concurrently with the other predictors. In addition to the left hippocampal volume, age, arteriopathy, and measures of behavioural control were identified as significant predictors of performance on LM. Only the left hippocampus and arteriopathy made a significant and unique contribution to retention on LM in the visual rating model, although this second model with fewer predictors explained a smaller portion of the variance in retention scores on LM.

It was also necessary to construct two final multivariate models for VR. Left hippocampal volume, age, estimated IQ, and the number of medications with potential cognitive effects taken were all found to make a significant contribution to performance in the first model. However, only visual ratings of the right hippocampus and estimated IQ were retained in the second VR model. Therefore, contrary to expectations, there was no consistent lateralised association between right hippocampal size and non-verbal memory function. Rather, the findings of the two models for VR were at odds with one another, with the first including the left 
Table 3 Final multivariate models for logical memory and visual reproduction

\begin{tabular}{|c|c|c|c|c|c|c|c|c|}
\hline \multirow[b]{3}{*}{ Predictors } & \multicolumn{4}{|c|}{ LM \% retention } & \multicolumn{4}{|c|}{ VR \% retention } \\
\hline & \multicolumn{2}{|l|}{ Model 1} & \multicolumn{2}{|c|}{ Model 2} & \multicolumn{2}{|l|}{ Model 1} & \multicolumn{2}{|c|}{ Model 2} \\
\hline & $\hat{\hat{\beta}}$ & $\mathbf{p}$ & $\overline{\hat{\beta}}$ & $\mathbf{p}$ & $\overline{\hat{\beta}}$ & $\mathbf{p}$ & $\overline{\hat{\beta}}$ & $\mathbf{p}$ \\
\hline Left HV & 2.79 & 0.000 & $\dagger$ & $\dagger$ & 2.25 & 0.001 & $\dagger$ & $t$ \\
\hline Right HV & & & $\dagger$ & $\dagger$ & * & * & $\dagger$ & $\dagger$ \\
\hline Left HVR & $\dagger$ & $\dagger$ & 10.01 & 0.000 & $\dagger$ & $\dagger$ & & \\
\hline Right HVR & $\dagger$ & $\dagger$ & & & $\dagger$ & $\dagger$ & 8.85 & 0.001 \\
\hline Sex & * & * & * & * & * & * & * & * \\
\hline Age & -1.86 & 0.009 & & & -1.85 & 0.033 & & \\
\hline Years of education & * & * & * & * & & & & \\
\hline Estimated IQ & * & * & * & * & 0.94 & 0.000 & 0.89 & 0.001 \\
\hline Timed walk & * & * & * & * & * & * & * & * \\
\hline Arteriopathy & 3.72 & 0.024 & 3.86 & 0.024 & * & * & * & * \\
\hline PVH frontal & * & * & * & * & * & * & * & * \\
\hline PVH occipital & * & * & * & * & * & * & * & * \\
\hline No. of meds & * & * & * & * & -7.03 & 0.035 & & \\
\hline Drive & * & * & * & * & * & * & * & * \\
\hline Control & -10.48 & 0.002 & & & * & * & * & * \\
\hline Hearing & * & * & * & * & * & * & * & * \\
\hline $\mathrm{R}^{2}$ & 0.33 & & 0.22 & & 0.26 & & 0.21 & \\
\hline$P$ & 0.000 & & 0.000 & & 0.000 & & 0.000 & \\
\hline$d f$ & 4 & & 2 & & 4 & & 2 & \\
\hline \multicolumn{9}{|c|}{$\begin{array}{l}\text { Models } 1 \text { and 2: if both the hippocampal volumes and visual ratings emerged as significant univariate predict } \\
\text { for the same measure, two alternate memory models were defined for the memory measure. } \\
\text { Empty cells, non-significant multivariate contribution; *non-significant univariate contribution; tvariable not } \\
\text { entered into model because of overlap with another predictor. } \\
\text { LM, logical memory; PVH, periventricular hyperintensities; VR, visual reproduction; HV, hippocampal volume, } \\
\text { HVR, hippocampal visual rating; meds, medications. }\end{array}$} \\
\hline
\end{tabular}

hippocampal volume and the second involving the visual rating of the right hippocampus.

It was only necessary to construct one multivariate model for CVLT per cent retention, as the volumetric hippocampal measures failed to reach univariate significance. Visual ratings of the left hippocampus, sex, age, estimated IQ, arteriopathy, and number of medications with potential cognitive effects were all identified as making a unique contribution to per cent retention scores on CVLT. This resulted in a robust model that accounted for almost half of the variance in scores on this measure.

Two multivariate models were defined for total learning on CVLT. The left hippocampal measures (volumetric and visual) were significantly associated with performance on this measure in their respective models. In both models, sex, age, estimated IQ, and measures of response drive also made a significant independent contribution to explaining variance in total learning scores on CVLT.

\begin{tabular}{|c|c|c|c|c|c|c|}
\hline \multirow[b]{3}{*}{ Predictors } & & & \multicolumn{4}{|c|}{ CVLT \% learning } \\
\hline & \multicolumn{2}{|c|}{ CVLT \% retention } & \multicolumn{2}{|l|}{ Model 1} & \multicolumn{2}{|c|}{ Model 2} \\
\hline & $\hat{\hat{\beta}}$ & $p$ & $\overline{\hat{\beta}}$ & $p$ & $\overline{\hat{\beta}}$ & $P$ \\
\hline Leff $\mathrm{HV}$ & * & * & 0.72 & 0.003 & $\dagger$ & $t$ \\
\hline Right HV & * & * & * & & $\dagger$ & \\
\hline Left HVR & 10.40 & 0.000 & $\dagger$ & $\dagger$ & 3.40 & 0.001 \\
\hline Right HVR & & & $\dagger$ & $\dagger$ & & \\
\hline Sex & -19.03 & 0.000 & -6.47 & 0.001 & -5.51 & 0.003 \\
\hline Age & -1.82 & 0.027 & -1.10 & 0.000 & -0.83 & 0.008 \\
\hline Years of education & * & * & * & * & * & \\
\hline Estimated IQ & 0.62 & 0.009 & 0.35 & 0.000 & 0.30 & 0.001 \\
\hline Timed walk & * & & & & & \\
\hline Arteriopathy & 5.43 & 0.004 & * & * & * & * \\
\hline PVH frontal & * & & & & & \\
\hline PVH occipital & & & * & * & * & * \\
\hline Number of medications & -8.63 & 0.008 & * & * & * & * \\
\hline Drive index & & & -2.92 & 0.001 & -1.98 & 0.016 \\
\hline Control index & * & * & & & & \\
\hline Hearing & * & * & & & & \\
\hline $\mathrm{R}^{2}$ & 0.47 & & 0.43 & & 0.44 & \\
\hline p & 0.000 & & 0.000 & & 0.000 & \\
\hline df & 6 & & 5 & & 5 & \\
\hline \multicolumn{7}{|c|}{$\begin{array}{l}\text { Models } 1 \text { and 2: if both the hippocampal volumes and visual ratings emerged as significant univariate predicto } \\
\text { for the same measure, two alternate memory models were defined for the memory measure. } \\
\text { Empty cells, non-significant multivariate contribution; }{ }^{*} \text { non-significant univariate contribution; tvariable not } \\
\text { entered into model because of overlap with another predictor. } \\
\text { CVLT, California Verbal Learning Test; PVH, periventricular hyperintensities; HV, hippocampal volume, HVR, } \\
\text { hippocampal visual rating. }\end{array}$} \\
\hline
\end{tabular}




\section{Hippocampal size and memory performance in successful ageing}

Compared with the whole sample, the subgroup with $\mathrm{CDR}=0$ did not differ significantly with respect to age, education, or estimated IQ, although they had significantly higher MMSE scores $(t=-2.95 ; \mathrm{df}=96 ; \mathrm{p}=0.004)$. In accordance with expectation, the subgroup with $\mathrm{CDR}=0$ achieved superior mean scores on all memory measures to those of the whole sample. Of note, however, even some of the successful ageing group failed to retain any information after a 30-minute delay on VR or CVLT. Moreover, the standard deviations observed on some measures reflected appreciable variability in retention scores even amongst those individuals not demonstrating cognitive decline.

The relationships between the memory measures and the hippocampal, sociodemographic, and medical predictors were re-examined in the reduced sample, who were considered to be unambiguously healthy. The final multivariate models for each memory measure for this subgroup with $\mathrm{CDR}=0$ are presented in table 5 . As in the previous tables, predictors that become non-significant on all memory measures following the univariate analyses were excluded from the table.

The left hippocampal volume was found to be the only significant predictor of scores on LM in successful ageing; larger volumes were associated with superior memory performance. None of the other variables from the subset of hippocampal, sociodemographic, or medical predictors reached univariate significance.

Estimated IQ was identified as the only variable with a significant and unique influence on per cent retention on VR. The visual and volumetric hippocampal measures failed to impact significantly upon performance of this task.

A number of variables were identified as significant univariate predictors of per cent retention scores on the CVLT, including visual ratings of the left and right hippocampi, sex, and periventricular hyperintensities in the occipital region. However, qualitative visual ratings of the left hippocampus were identified as the only significant predictor on this score when the influence of the predictors was examined simultaneously in a multivariate context.

The acquisition of words on the learning trials $1-5$ of the CVLT was significantly affected by sex, estimated IQ, and hearing deficits. Females outperformed males on this task in this cognitively healthy group. Left hippocampal volumes failed to maintain significance once the impact of these other predictors was taken into account.

\section{DISCUSSION}

One of the major findings of this study is that left hippocampal size, estimated by either visual or volumetric means, is an important determinant of per cent retention scores on a verbal memory task (LM) in community dwelling individuals over 80 years of age. The fact that the visual measures proved to be as sensitive as the volumetric measures in many cases has important clinical implications for the effectiveness of this simple and efficient clinical technique, as visual ratings are typically the method of choice for examining MRI scans because more complex and technically demanding volumetrics are not generally available for routine clinical use.

The left hippocampal measures were identified as robust predictors of delayed retention, even when forced to compete against an extensive array of medical and sociodemographic predictors. This finding supports previous reports of a correlation between hippocampal size and delayed paragraph recall in healthy older persons, ${ }^{25}{ }^{26}$ and highlights the crucial role the left hippocampus plays in delayed retention of verbal material. Moreover, the left hippocampal volume was the only significant predictor of retention on LM in the successful ageing subgroup with $\mathrm{CDR}=0$, providing further support for a direct link between memory and hippocampal function by showing that the relationship is observed even in the absence of cognitive decline.

A similar finding was reported by Petersen et al, ${ }^{27}$ who observed that the left hippocampus was predictive of delayed recall on $\mathrm{LM}$ in $\mathrm{AD}$ patients. However, these researchers failed to identify hippocampal volumes as a significant predictor of LM performance in a group of healthy elderly controls (average age 79.1 years), which they attributed to insufficient variability in hippocampal volumes in normal ageing. It is possible that our normal group was more heterogeneous because of their more advanced age, which may account for these discrepant findings. Nevertheless, in keeping with the current results, significant positive correlations between MRI measures of the left hippocampus and retention on LM have been reported in studies of epilepsy ${ }^{28} 29$ and brain injury. ${ }^{7}$

Age was found to have a significant impact on LM, which is consistent with the general consensus in the literature that memory function is adversely affected by advancing age. ${ }^{30-33}$ However, this study extends previous findings as it implies that age influences performance on this task over and above the influence of age related illnesses per se. Along similar lines, Jack et $a l^{34}$ speculated that brain changes associated with age related medical conditions may have accounted for some of their observed correlation between age

Table 5 Final models for the memory measures in the subgroup with $C D R=0$

\begin{tabular}{|c|c|c|c|c|c|c|c|c|}
\hline \multirow[b]{2}{*}{ Predictors } & \multicolumn{2}{|c|}{ LM \% retention } & \multicolumn{2}{|c|}{ VR \% retention } & \multicolumn{2}{|c|}{ CVLT \% retention } & \multicolumn{2}{|c|}{ CVLT total learning } \\
\hline & $\hat{\boldsymbol{\beta}}$ & $p$ & $\hat{\boldsymbol{\beta}}$ & $p$ & $\hat{\hat{\boldsymbol{\beta}}}$ & $p$ & $\overline{\hat{\beta}}$ & $p$ \\
\hline Left $\mathrm{HV}$ & 1.99 & 0.005 & * & * & * & * & * & * \\
\hline Right $\mathrm{HV}$ & $*$ & $*$ & * & * & * & * & * & * \\
\hline Leff HVR & * & * & * & * & 9.75 & 0.004 & & \\
\hline Right HVR & * & * & * & * & & & * & * \\
\hline Sex & * & * & * & * & & & -8.01 & 0.000 \\
\hline Years of education & * & * & & & * & * & * & * \\
\hline Estimated IQ & * & * & 1.16 & 0.001 & * & * & 0.31 & 0.006 \\
\hline PVH occipital & * & * & * & * & & & * & * \\
\hline Hearing & * & * & * & * & * & * & -3.16 & 0.026 \\
\hline $\mathrm{R}^{2}$ & 0.13 & & 0.19 & & 0.14 & & 0.35 & \\
\hline$p$ & 0.005 & & 0.001 & & 0.004 & & 0.000 & \\
\hline DF & 1 & & 1 & & 1 & & 3 & \\
\hline
\end{tabular}

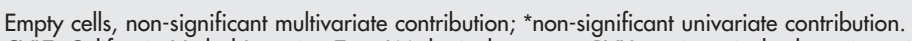
CVLT, California Verbal Learning Test; LM, logical memory; PVH, periventricular hyperintensities; HV, hippocampal volume, HVR, hippocampal visual rating; VR, visual reproduction. 
and volume loss in normal ageing. In the current study, arteriopathy and scores on the behavioural control index significantly impacted upon scores on LM, indicating that some markers of general illnesses associated with ageing do influence memory performance. However, the extrapyramidal measures and the white matter ratings failed to impact significantly upon performance on any of the memory tasks.

A higher arteriopathy score was associated with better retention on LM. This was unexpected. One possible explanation for this finding could be that individuals with poorer memory function may have under-reported the presence of vascular risk factors. This is supported by the fact that the association disappeared when the arteriopathy score was based on objective medical data rather than self report.

The identification of the visual ratings of the right hippocampus as a significant predictor of retention on VR is consistent with the notion of lateralisation of function, and is in keeping with previous studies that have documented an association between right hippocampal size and non-verbal memory performance. ${ }^{27}{ }^{35}$ In contrast, the hippocampal volumes showed the reverse pattern, with the left, but not the right, volume significantly contributing to retention on this task. This pattern has been reported by others, ${ }^{7}$ and has prompted speculation that the nature of the figures may permit some verbal encoding, thereby resulting in some contribution from the left hemisphere ${ }^{36}$ and a less lateralised effect. Alternatively, this inconsistent finding across the visual and volumetric measures of hippocampal size might be better explained by the high correlations between the left and right measures for each assessment technique (correlation coefficients were 0.77 and 0.97 for the volumetric and visual measures respectively). Therefore, in each case, either the left or right measure may have been retained in the final model owing to only a slight statistical difference relative to its counterpart. In support of this interpretation, when the final VR models were re-run by entering the contralateral measures to those that were actually retained (that is, left visual rating, right hippocampal volume), this had a negligible effect on the results. This indicates that there was no compelling evidence of a clear laterality effect for VR per cent retention.

Estimated IQ was a robust predictor of per cent retention on VR. It appears that general intellectual functioning plays a major role in influencing performance on this task, although no such association was identified for LM. Age was also identified as an influential predictor of retention on VR, but only in the model associated with the hippocampal volumes. Of the "other" medical predictors, only medications had a significant bearing on VR. This finding highlights the potentially deleterious impact of some medications on neuropsychological performance. Further, it is in keeping with the commonly held view that the human memory system is particularly susceptible to impairment associated with the long term use of several drugs that may be prescribed to treat a range of common illness in old age, such as anticholinergics and benzodiazepines. ${ }^{37}$

Visual ratings of the left hippocampus were a powerful predictor of per cent retention on CVLT in both the whole sample and the subset with no evidence of cognitive decline. This finding is in broad agreement with previous demonstration of a significant association between left hippocampal volumes and CVLT delayed recall in a combined group of elderly control subjects and $\mathrm{AD}$ patients. ${ }^{27}$ However, it remains unclear as to why the hippocampal volumes were not themselves sensitive to performance on this task in the current investigation.

On average, women recalled 19\% more information than men on CVLT. Such a gender effect, with women outperforming men, has previously been reported on a range of memory measures, ${ }^{26}{ }^{38}$ including learning and delayed recall on the CVLT. ${ }^{39}$ In keeping with LM, a similar age effect was observed on CVLT, as well as an association between arteriopathy score and memory performance. While the underlying reason for this latter finding remains unclear, it may be unique to retention of verbal material, as arteriopathy failed to remain in the model for the only non-verbal task, and also did not affect acquisition of verbal information.

Left hippocampal size was predictive of learning on the CVLT, irrespective of whether this was measured by visual or volumetric means. Hippocampal volumes have previously been shown to correlate significantly with learning on the CVLT. ${ }^{27}$ The specific contribution of the left hippocampal measures to performance on this verbal task is consistent with the notion of hemispheric lateralisation of function. The gender effect again signified a female advantage in terms of learning scores on CVLT. Age, estimated IQ, and response drive also had a robust and independent influence on encoding of items on CVLT. This latter finding may be due to the fact that the participants reported that this task was particularly demanding, and consequently any subject with decreased drive may have been less willing than others to persist on this task.

Hippocampal measures were not identified as important contributors to performance on visual reproduction or total learning on the CVLT in successful ageing. Instead, these memory measures were influenced by sociodemographic and medical predictors. In the successful ageing group, hippocampal measures only contributed to performance on tasks measuring delayed retention of verbal information. Such tasks may be more heavily reliant on hippocampal integrity than other memory tasks, or these verbal memory measures may be more specific tests of hippocampal function. Nevertheless, the analyses carried out on the reduced sample demonstrate that the association between hippocampal size and memory performance on certain tasks is robust enough to manifest in a group of optimally healthy older persons, even after sociodemographic and medical variables are taken into account.

In summary, the current findings reinforce the widely held notion that the hippocampus plays an intimate role in the neural processing of memory. The importance of these results, however, is that they extend on previous findings by delineating the specific role of the hippocampus in predicting memory functioning in community dwelling seniors from the oldest segment of the population. Hippocampal volumes and visual assessment of the hippocampus are among the strongest predictors of memory performance in normal ageing, even when the influence of age related disease and sociodemographic variables is accounted for. The robustness of this relationship was further substantiated by demonstration of a strong association between memory and hippocampal size in successful ageing, which indicates that hippocampal size is an important predictor of memory performance even in individuals who remain cognitively well into their ninth and tenth decades of life.

The current findings are indicative of a genuine relation between the hippocampus and memory performance that is not simply mediated by the onset of age related disease, but is also upheld in a group of older persons who are ageing successfully. Nevertheless, it remains unclear as to whether this association can be explained in terms of an underlying pathological entity such as a preclinical Alzheimer's-type process, or whether the relation can be accounted for within a model of normal ageing. It is possible that those individuals with poorer memory performance may have always had smaller hippocampi, thus explaining the observed 
association. Alternatively, the relation between hippocampal size and memory function may arise from hippocampal atrophy coinciding with memory decline in some individuals. The ongoing study of these participants in the Sydney Older Persons Study should help to clarify this issue by providing information about any changes that may occur in the memory performance or hippocampal size of these individuals in a longitudinal context.

\section{ACKNOWLEDGEMENTS}

This research was supported in part by a grant from the National Health and Medical Research Council of Australia, an Infrastructure Stream C grant from the New South Wales Department of Health, Australia, and by the Ageing and Alzheimer's Research Foundation.

\section{Authors' affiliations}

T C Lye, O Piguet, H Creasey, Centre for Education and Research on Ageing, Concord Hospital, Sydney, Australia

O Piguet, H P Bennett, G A Broe, Prince of Wales Medical Research Institute and the University of New South Wales, Sydney, Australia D A Grayson, School of Psychology, the University of Sydney, Sydney, Australia

L J Ridley, Department of Radiology, Concord Hospital, Sydney, Australia

G Anthony Broe, Prince of Wales Hospital, Randwick, Sydney, Australia Competing interests: none declared

\section{REFERENCES}

1 Scoville WB, Milner B. Loss of recent memory after bilateral hippocampal lesions. J Neurol Neurosurg Psychiatry 1957;20:11-21.

2 Gadian DG, Mishkin M, Vargha-Khadem F. Early brain pathology and its relation to cognitive impairment: the role of quantitative magnetic resonance techniques. Adv Neurol 1999;81:307-15.

3 Wood AG, Saling MM, O'Shea MF, et al. Components of verbal learning and hippocampal damage assessed by T2 relaxometry. J Int Neuropsychol Soc 2000;6:529-38.

4 Golomb J, Kluger A, de Leon MJ, et al. Hippocampal formation size predicts declining memory performance in normal aging. Neurology 1996;47:810-13.

5 Cahn DA, Sullivan EV, Shear PK, et al. Structural MRI correlates of recognition memory in Alzheimer's disease. J Int Neuropsychol Soc 1998;4:106-14.

6 Deweer B, Lehéricy S, Pillon B, et al. Memory disorders in probable Alzheimer's disease: the role of hippocampal atrophy as shown with MRI. J Neurol Neurosurg Psychiatry 1995;58:590-7.

7 Bigler ED, Johnson SC, Anderson CV, et al. Traumatic brain injury and memory: The role of hippocampal atrophy. Neuropsychology 1996; 10:333-42

8 Waite LM, Broe GA, Grayson DA, et al. The incidence of dementia in an Australian community population: the Sydney Older Persons Study. Int J Geriatr Psychiatry $2001 ; 16: 680-9$

9 Waite LM, Broe GA, Grayson DA, et al. Preclinical syndromes predict dementia: the Sydney older persons study. J Neurol Neurosurg Psychiatry 2001;71:296-302.

10 Hughes CP, Berg L, Danziger WL, et al. A new clinical scale for the staging of dementia. Br J Psychiatry 1982;140:566-72.

11 Wechsler D. WMS-R manual. San Antonio, TX: The Psychological Corporation, 1987

12 Delis DC, Kramer JH, Kaplan E, et al. California verbal learning test: adult version manual. San Antonio, TX: The Psychological Corporation, 1987.

13 Cook MJ, Fish DR, Shorvon SD, et al. Hippocampal volumetric and morphometric studies in frontal and temporal lobe epilepsy. Brain 1992;115:1001-15.
14 Desmond PM, O'Brien JT, Tress BM, et al. Volumetric and visual assessment of the mesial temporal structures in Alzheimer's disease. Aust NZ J Med 1994;24:547-53.

15 Piguet O, Ridley L, Grayson DA, et al. Are MRI white matter lesions clinically significant in the 'old-old'? Evidence from the Sydney Older Persons Study. Dement Geriatr Cogn Disord 2003;15:143-50.

16 Piguet O, Grayson D, Broe GA, et al. Normal aging and executive functions in "old-old" community dwellers: Poor performance is not an inevitable outcome. Int Psychogeriatr 2002;14:139-59.

17 Scheltens $P$, Barkhof $F$, Leys $D$, et al. A semiquantitative rating scale for the assessment of signal hyperintensities on magnetic resonance imaging. J Neurol Sci 1993;114:7-12.

18 Scheltens $\mathbf{P}$, Erkinjuntti $T$, Leys $\mathrm{D}$, et al. White matter changes on $\mathrm{CT}$ and MRI: an overview of visual rating scales. Eur Neurol 1998;39:80-9.

19 Nelson HE, Willison J. National adult reading test (NART): test manual. Windsor, UK: NFER Nelson, 1991.

20 Gunning-Dixon FM, Raz N. The cognitive correlates of white matter abnormalities in normal aging: A quantitative review. Neuropsychology 2000;14:224-32.

21 Radloff LS. The CES-D scale: a self-report depression scale for research in the general population. App Psychol Meas 1977;1:385-401.

22 Neugarten BI, Havinghurst RJ, Tobin SS. The measurement of life satisfaction. $J$ Gerontol 1961;16:134-43.

23 Elsass L, Kinsella G. Development of a scale for measuring behavioural change following closed head injury. In: Anderson V, Bailey M, eds. Theory and function - bridging the gap: proceedings of the fourteenth annual brain impairment conference. Melbourne: Australian Society for the Study of Brain Impairment, 1989:124-31.

24 Waite L, Grayson D, Jorm AF, et al. Informant-based staging of dementia using the Clinical Dementia Rating. Alzheimer Dis Assoc Disord $1999 ; 13: 34-7$

25 de Leon MJ, George AE, Golomb J, et al. Frequency of hippocampal formation atrophy in normal aging and Alzheimer's disease. Neurobiol Aging 1997;18:1-11.

26 Golomb J, Kluger A, de Leon MJ, et al. Hippocampal formation size in normal human aging: A correlate of delayed secondary memory performance. Learn Mem 1994; 1:45-54.

27 Petersen RC, Jack CR Jr, Xu YC, et al. Memory and MRI-based hippocampal volumes in aging and AD. Neurology 2000;54:581-7.

28 Lencz T, McCarthy G, Bronen RA, et al. Quantitative magnetic resonance imaging in temporal lobe epilepsy: Relationship to neuropathology and neuropsychological function. Ann Neurol 1992;31:629-37.

29 Martin RC, Hugg JW, Roth DL, et al. MRI extrahippocampal volumes and visual memory: Correlations independent of MRI hippocampal volumes in temporal lobe epilepsy patients. J Int Neuropsychol Soc 1999;5:540-8.

30 Crook T, Bartus RT, Ferris SH, et al. Age-associated memory impairment: Proposed diagnostic criteria and measures of clinical change-Report of a National Institute of Mental Health work group. Dev Neuropsychol 1986;2:261-76.

31 Ebly EM, Hogan DB, Parhad IM. Cognitive impairment in the nondemented elderly: Results from the Canadian Study of Health and Aging. Arch Neurol 1995;52:612-19.

32 Hänninen T, Soininen H. Age-associated memory impairment. Normal aging or warning of dementia? Drugs \& Aging 1997;11:480-9.

$33 \mathrm{Kral}$ VA. Senescent forgetfulness: Benign and malignant. Can Med Assoc J 1962;86:257-60.

34 Jack CR Jr, Petersen RC, Xu YC, et al. Medial temporal atrophy on MRI in normal aging and very mild Alzheimer's disease. Neurology 1997:49:786-94.

35 Mori E, Yoneda Y, Yamashita H, et al. Medial temporal structures relate to memory impairment in Alzheimer's disease: An MRI volumetric study. J Neurol Neurosurg Psychiatry 1997;63:214-21.

36 Lezak MD. Neuropsychological assessment. New York: Oxford University Press, 1995.

37 Selkoe DJ. Aging brain, aging mind. Sci Am 1992;267:135-42.

38 Larrabee GJ, Crook TH. Do men show more rapid age-associated decline in simulated everyday verbal memory than do women? Psychol Aging 1993;8:68-71

39 Kramer JH, Delis DC, Daniel M. Sex differences in verbal learning. J Clin Psychol 1988;44:907-15 